\title{
WHICH ABELIAN GROUPS CAN BE FUNDAMENTAL GROUPS OF REGIONS IN EUCLIDEAN SPACES ? ${ }^{1}$
}

\author{
BY BAI CHING CHANG
}

Communicated by Emery Thomas, November 10, 1971

Let $C_{n}$ denote the collection of all abelian groups that can be fundamental groups of regions in $S^{n}$. It is clear that $C_{k} \subseteq C_{k+1}$. It is also easy to see that $C_{1}$ and $C_{2}$ each consist of just two groups-the trivial groups 1 and the infinite cyclic group $Z$. We shall see in this paper that actually $C_{k}=C_{k+1}$ for $k \geqq 4$, so we shall be concerned mainly with the difference between regions in $S^{3}$ and regions in $S^{4}$.

If a region $A$ in $S^{n}$ is not $S^{n}$ itself, we may assume that $A \subset R^{n}$, and that there is a point $e$ of $A$ that is at a distance $\geqq 1$ from $R^{n}-A$. Using barycentric subdivision $T_{k}$ of $R^{n}$ of mesh converging to zero, where $T_{l}$ is a refinement of $T_{k}$ if $l<k$, let $U_{k}$ be the interior of the union of those simplexes that lie in $A$ and are at a distance $\leqq k$ from $e$. Take $A_{k}$ to be the component of $U_{k}$ that contains $e_{i}$. It is easy to see that $A_{l} \leqq A_{k}$ if $l<k$, and that $\bigcup_{k=1}^{\infty} A_{k}=A$; thus $\pi(A)$ is equal to the direct limit of the sequence $\left\{\pi\left(A_{k}\right)\right\}$. Since each $\pi\left(A_{k}\right)$ is finitely generated, $\pi(A)$ must be countable.

Now suppose that $G=\pi(A)$ is abelian. Since $G_{i}=\pi\left(A_{i}\right)$ is finitely generated, the image $K_{i}$ of $G_{i}$ in some $G_{s}=\pi\left(A_{s}\right)$ of the inclusion $G_{i} \rightarrow G_{s}$ must be abelian. Replacing the sequence $\left\{G_{i}\right\}$ by a subsequence if necessary, we may assume that the image $K_{i}$ of $G_{i}$ in $G_{i+1}$ is abelian.

The calculation of $C_{3}$ is closely related to the following problem: "Which elements of a link group commute?" In fact, if we use brick subdivision instead of barycentric subdivision of $R^{3}$ in the construction of $A_{k}$, we may assume that each $S^{3}-A_{k}$ is the union of a finite number of handle-bodieswith-knotted-holes, semilinearly imbedded in $S^{3}$. Since each $G_{k}$ is finitely generated, so is its abelianized group $\bar{G}_{k}=H_{1}\left(A_{k}\right)$. We can find nonsingular loops $\left\{x_{1}, \ldots, x_{p}\right\}$ that generate $H_{1}\left(A_{k}\right)$. By the Alexander duality theorem and the fact that $S^{3}-A_{k}$ is a manifold, we can also find nonsingular loops $\left\{y_{1}, \ldots, y_{p}\right\}$ in $S^{3}-A_{k}$ which are dual to $\left\{x_{1}, \ldots, x_{p}\right\}$ in the sense that the linking number $\left(x_{i}, y_{i}\right)$ between $x_{i}$ and $y_{i}$ is equal to $\delta_{i j}$, where $\delta_{i j}$ is the Kronecker delta. The image of any two elements of $G_{k-1}$ in $G_{k}$ must commute in the complement of the link $y_{1} \cup y_{2} \cup \cdots \cup y_{p}$.

The following theorem (cf. [6] and [7]) makes it possible to deal with arbitrary links.

AMS 1969 subject classifications. Primary 5520, 5705.

${ }^{1}$ This paper represents a portion of the author's Ph.D. thesis, written under the direction of Professor Ralph H. Fox at Princeton University.

Copyright (C) American Mathematical Society 1972 
THEOREM A. Let l be a link with two components whose linking number is $\lambda$. Then $G$, the group of $l, \bmod G_{2}$, its second lower central subgroup, has the following presentation: $\left\{a, b:\left[a, b^{\lambda}\right]=\left[a^{\lambda}, b\right]=1, F_{2}=1\right\}$, where $F$ is the free group $\{a, b\}$.

Now with the help of this theorem and free calculus (cf. [2]) we can prove the following theorem:

THEOREM 1. Let $l_{1}, l_{2}, \ldots, l_{n}$ be components of $a$ link $l$ and let $a_{1}, a_{2}, \ldots, a_{n}$ be their meridians. If two elements $x$ and $y$ in $\pi\left(S^{3}-l\right)$ commute, and if ${ }^{2}$ $x \sim a_{1}^{\alpha_{1}} a_{2}^{\alpha_{2}} \ldots a_{n}^{\alpha_{n}}, y \sim a_{1}^{\beta_{1}} a_{2}^{\beta_{2}} \ldots a_{n}^{\beta_{n}}$, then the linking number $\lambda_{i j}$ of $l_{i}$ and $l_{j}$ must divide

$$
\left|\begin{array}{cc}
\alpha_{i} & \alpha_{j} \\
\beta_{i} & \beta_{j}
\end{array}\right|
$$

To calculate $C_{3}$ we also need the following theorem:

THeOREM 2. If $G$ is the fundamental group of a region $A$ in $S^{3}$, then no abelian subgroup of $G$ has rank greater than 2 .

The proof of Theorem 2 is merely a modification of an argument due to Conner (cf. [1, Theorem 2]). By Theorem 2 we know that each $K_{m}$ must be either $1, Z$, or $Z+Z$. We can now state our theorem about regions in $S^{3}$ :

THEOREM 3. An abelian group $G$ is the fundamental group of a region $A$ in $S^{3}$ if and only if $G$ is $1, Z, Z+Z$, or a subgroup of the additive group of rational numbers.

It is not difficult to prove the "if" part of Theorem 3. The trivial knot has $Z$ as its group, and the trivial link whose linking number is 1 (see Figure 1) has $Z+Z$ as its fundamental group, and the well-known $P$-adic

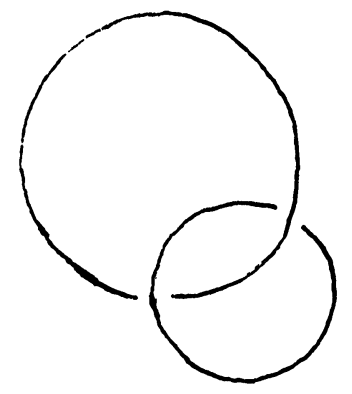

FIGURE 1

solenoid (cf. [3]) gives us the $P$-adic group as the fundamental group of its complement. The complements of other kinds of solenoids give us regions

\footnotetext{
${ }^{2}$ We write $f \sim g$ to denote that $f$ is homologous to $g$.
} 
whose fundamental groups are just the various subgroups of the additive group of rational numbers (cf. [5, Chapter VIII]). For the "only if" part, we need Theorem 1 and Theorem 2, and some other constructions and lemmas. It is too long to give here.

Before we go on to find $C_{4}$, it is interesting to see some results about a union of knotted 2-spheres in $S^{4}$. We have the following theorem, analogous to Theorem A.

THEOREM 4. For a disjoint union of an arbitrary number of spheres in $S^{4}$, the group $G=\pi\left(S^{4}-S_{1} \cup \cdots \cup S_{n}\right)$ depends $\bmod G_{2}$ only on the number of spheres. In fact $G / G_{2}=F / F_{2}$, where $F$ is the free group with $n$ generators. ${ }^{3}$

Therefore except for 1 and $Z$, no abelian group can be the group $G$. Furthermore, the center $C$ of $G$ must be contained in the commutator subgroup $G_{1}$ of $G$.

The proof of Theorem 4 makes use of the method of hyperplane crosssection (cf. [4]). We can represent the imbedding of $S_{1} \cup S_{2} \cup \ldots \cup S_{n}$ by a family of links $l_{1}^{t} \cup l_{2}^{t} \cup \cdots \cup l_{n}^{t}$, where $l_{i}^{t}$ is the cross section of $S_{i}$ with the hyperplane $x_{4}=t$, where $\left(x_{1}, x_{2}, x_{3}, x_{4}\right)$ is the coordinate of a point in $R^{4}$. The proof needs the fact that the presentation given in Theorem $A$ is almost canonical, and also the following theorem:

THEOREM 5 . The linking number $\left(K_{i}^{t}, l_{j}^{t}\right)$ between a component $K_{i}^{t}$ of $l_{i}^{t}$ with the totality of $l_{j}^{t}$ is always zero. ${ }^{4}$

Theorem 5 has its own interest, in the sense that it gives us a necessary condition for a link to be a link sliced from $n 2$-spheres. In general in order that $l$ be a slice of a union of $n 2$-spheres, we must be able to orient $l$ and divide $l$ into $n$ links $l_{1} \cup l_{2} \cup \cdots \cup l_{n}$ in such a way that the linking number of any component of $l_{i}$ with $l_{j}$ for any $i \neq j$ is always zero.

Now we may state our theorem about regions in $S^{4}$.

THEOREM 6. An abelian group $G$ is the fundamental group of a region in $S^{n}$ for $n \geqq 4$ if and only if it is countable.

By what we said at the beginning of this paper, it is clear that we need only to prove the "if" part for the case $n=4$, and it follows that $C_{4}=C_{5}=\cdots$. The only way to prove this theorem is to give an algorithm to construct a region $A$ in $S^{4}$ (actually in $R^{4}$ ) whose fundamental group is a given countable abelian group $G$. It is too long to give here.

\section{REFERENCES}

1. P. E. Conner, On the action of a finite group on $S^{n} \times S^{n}$, Ann. of Math. (2) 66 (1957), 586-588. MR 20 \# 2725.

\footnotetext{
${ }^{3}$ We assume our imbedding is semilinear and locally flat.

${ }^{4}$ We assume that $S_{i}$ are orientated, thus orientation is induced on each $l_{i}$.
} 
2. R. H. Crowell and R. H. Fox, Introduction to knot theory, Ginn, Boston, Mass., 1963. MR 26 \# 4348.

3. S. Eilenberg and N. E. Steenrod, Foundations of algebraic topology, Princeton Univ. Press, Princeton, N.J., 1952. MR 14, 398.

4. R. H. Fox, A quick trip through knot theory, Topology of 3-Manifolds and Related Topics (Proc. The Univ. of Georgia Inst., 1961), Prentice-Hall, Engelwood Cliffs, N.J., 1962, pp. 120-167. MR 25 \#3522.

5. A. G. Kuroš, Theory of groups, GITTL, Moscow, 1953; English transl., vol. II, Chelsea, New York, 1955. MR 15, 501 ; MR 17, 124.

6. J. W. Milnor, Link groups, Ann. of Math. (2) 59 (1954), 177-195. MR 17, 70.

7. - Isotopy of links, Algebraic Geometry and Topology, Princeton Univ. Press, Princeton, N.J., 1957, pp. 280-306. MR 19, 1070.

Department of Mathematics, Princeton University, Princeton, New Jersey 08540 